\title{
Exclusion Problems and the Cardinality of Logical Space
}

\author{
Tim Button ${ }^{1}$ (D)
}

Received: 17 January 2016 / Accepted: 6 September 2016 / Published online: 4 October 2016 (C) The Author(s) 2016. This article is published with open access at Springerlink.com

\begin{abstract}
Wittgenstein's atomist picture, as embodied in his Tractatus, is initially very appealing. However, it faces the famous colour-exclusion problem. In this paper, I shall explain when the atomist picture can be defended (in principle) in the face of that problem; and, in the light of this, why the atomist picture should be rejected. I outline the atomist picture in Section 1. In Section 2, I present a very simple necessary and sufficient condition for the tenability (in principle) of the atomist picture. The condition is: logical space is a power of two. In Sections 3 and 4, I outline the colour-exclusion problem, and then show how the cardinality-condition supplies a response to exclusion problems. In Section 5, I explain how this amounts to a distillation of a proposal due to Moss (2012), which goes back to Carruthers (1990: 144-7). And in Section 6, I show how all this vindicates Wittgenstein's ultimate rejection of the atomist picture. The brief reason is that we have no guarantee that there are any solutions to a given exclusion problem but, if there are any, then there are far too many.
\end{abstract}

Keywords Atomist picture $\cdot$ Logical space $\cdot$ Colour-exclusion $\cdot$ Color incompatibility · Logical independence · Tractarian metaphysics · Ludwig Wittgenstein · Tractatus Logico-Philosophicus · Sarah Moss

Tim Button

button@cantab.net

1 University of Cambridge, Cambridge, UK 


\section{Wittgenstein's Atomist Picture}

Wittgenstein's atomist picture provides us with an extremely elegant, combinatorial picture of modality. Possibilities are generated by combinations of atomic propositions, which are in turn generated by combinations of simples. In what follows, I shall think of the possibilities as points in a logical space. (We can equally call these possibilities 'possible words', and call logical space 'the possible universe'; nothing will turn on this terminology).

Here is the atomist picture in more detail. The atomic propositions have a particular form (Wittgenstein [10]: 2-2.0212, 4.22)

Simples-Recombination. Atomic propositions consist of concatenations of (names for) simples. Subject only to constraints on their logical types, any arbitrary recombination of simples yields an atomic proposition.

So: given an atomic proposition, $a$, replacing the (names for) simples in $a$ with (names for) other simples of the same logical type yields another atomic proposition. These atomic propositions then generate the possibilities in logical space, via two principles ([10]: 4.211, 4.26-4.28, 5.134):

Modal-Valuationism. Each possibility in logical space uniquely corresponds to a valuation of the atomic propositions. A valuation assigns either truth or falsity (never both) to each atomic propositions.

Atomic-Independence. The atomic propositions are logically independent from one another: no assignment of a truth value to any atomic propositions determines the truth values of any other atomic propositions.

So: any combinatorial possible assignment of truth values to atomic propositions yields a possibility. These three principles record the atomist picture. And it provides an elegantly simple of logical space. It would be lovely, if it were true.

\section{An Elementary Cardinality-constraint}

I have described the atomist picture as combinatorial. In fact, it is intimately connected to an elementary cardinality-constraint. Stated briefly, this constraint is: logical space is a power of two. I use this phrase, to abbreviate the following claim: for some cardinal $\mathfrak{a}$, the cardinality of logical space is $2^{\mathfrak{a}}$, i.e. there are exactly $2^{\mathfrak{a}}$ possibilities. Note that I shall not insist that $\mathfrak{a}$ is finite; indeed, transfinite values of $\mathfrak{a}$ are probably to be expected.

Our cardinality-constraint is obviously a necessary condition for the tenability of the atomist picture, i.e.:

If logical space can be described by the atomist picture, then logical space is a power of two.

I say that this is obvious, because the atomist picture is so closely tied to the idea of truth tables (Wittgenstein [10]: 4.26-4.31, 4.442, [11]: 170-1; Ramsey [7]: 470), and 
a truth table with $\mathfrak{a}$ atomic propositions has exactly $2^{\mathfrak{a}}$ lines. But it is worth running through this point in detail, not least to ensure that it holds for 'infinite' truth tables (that is, when $\mathfrak{a}$ is infinite).

Suppose, then, that logical space can be described by the atomist picture. So we have some set, $A$, of atomic propositions. These propositions must each have exactly one truth value, by Modal-Valuationism, and their truth values are logically independent, by Atomic-Independence. So the number of possible complete assignments of truth/falsity to the atomic propositions is just the number of distinct functions from $A$ to the set $\left\{\right.$ True, False\}. Where $A$ 's cardinality is $\mathfrak{a}$, there are exactly $2^{\mathfrak{a}}$ such functions. So, by Modal-Valuationism, there are $2^{\mathfrak{a}}$ possibilities, as required.

In fact, this necessary condition is also a sufficient condition on the in-principle tenability of the atomist picture. That is:

If logical space is a power of two, then logical space can be described by the atomist picture.

To show this, I shall assume that logical space is a power of two, and use this assumption to construct a toy model which satisfies the atomist picture.

Suppose, then, that there are $2^{\mathfrak{a}}$ possibilities. Let $S$ be any set with cardinality $\mathfrak{a}$, and call S's members Switches. Every Switch is a simple. There is one further simple, Flicked. The atomic propositions are now all and only the concatenations of (the name of) Flicked together with (the name of) any Switch. Call the set of atomic propositions $A$. Call each $v \in \wp(A)$ a valuation; if $a \in v$, say that $v$ makes a true; if $a \notin v$, say that $v$ makes a false.

It remains to show that this toy model satisfies the atomist picture.

Concerning Simples-Recombination. The atomic propositions consist of any arbitrary concatenation of (names of) simples, subject to a constraint on their logical type which we can think of as follows: Flicked is a one-place property and the Switches are just first-order objects. ${ }^{1}$

Concerning Modal-Valuationism. We are treating $\wp(A)$ as the set of all possible valuations. Clearly both $A$ and $S$ have cardinality $\mathfrak{a}$, so that the cardinality of $\wp(A)$ is $2^{\mathfrak{a}}$. Since we assumed that there are also $2^{\mathfrak{a}}$ possibilities, there is a bijection between the set of possibilities and the set of valuations, i.e. every possibility uniquely corresponds to a valuation. Moreover, each valuation $v \in \mathcal{P}(A)$ says, of each atomic proposition in $A$, either that it is true, or that it is false, but never both.

Concerning Atomic-Independence. We are treating any $v \in \wp(A)$ as a valuation, i.e. an assignment of truth/falsity to the atomic propositions. So any combinatorially independent assignment of truth/falsity to the atomic propositions is legitimate.

\footnotetext{
${ }^{1}$ This follows Potter ([6]:232-3), who holds that Tractarian simples can be universals (see also Canfield [2]). This view has been controversial among Tractatus scholars; for example, Carruthers ([3]: 6-8, 137) explicitly rejects it. So, Carruthers would have to regard the atomic propositions, in this analysis, simply as the individual names for Switches (and nothing else). However, Carruthers is also a little hesitant about thinking that propositions might consist of just a single name. So Carruthers might deny that this kind of analysis is admissible. Nonetheless, in the cases which will matter most-where $\mathfrak{a}$ is infinite-there will be an abundance of toy analyses which would satisfy Carruthers; see Section 6.
} 
As such, our toy model satisfies all three principles of Wittgenstein's atomist picture, thereby establishing the sufficient condition. So we now obtain an elementary necessary and sufficient condition for the in-principle tenability of the atomist picture:

Logical space can be described by the atomist picture iff logical space is a power of two.

\section{Exclusion Problems}

I have set out the atomist picture, and shown how its combinatorial nature naturally leads to an elementary cardinality-constraint. I now want to outline the famous colour-exclusion problem. This was raised against Atomic-Independence by Wittgenstein himself ([10]: 6.3751, [11, 13]: 67-8).

Apparently, ${ }^{2}$ the proposition that $O$ is red and the proposition that $O$ is blue cannot both be true together: different colours exclude one another. So, if atomic propositions are logically independent from one another, as Atomic-Independence states, then colour-ascriptions cannot be atomic propositions. But colour-ascriptions seem like excellent candidates for atomic propositions. The example gives the problem its name, the colour-exclusion problem.

It is worth emphasising that colour-exclusion is just one example of an exclusion problem. Other examples are legion. No region can have multiple different temperatures; no object can have multiple different locations, masses, volumes or velocities; and so forth ([10]: 6.3751). More generally, exclusion arises whenever a property can come by degree $([11,12]$ VIII.91). Indeed, one might well say that exclusions are not the exception but the 'rule' ([13]: 64).

Faced with these exclusion problems, Wittgenstein ultimately abandoned AtomicIndependence ([10]: 167-9, [11]: VIII.78-84, [12]: 63-4, 74-6). I shall argue that he was right to do so, and for reasons which are intimately connected with the elementary cardinality-constraint outlined in Section 2. Crudely stated, the cardinalityconstraint allows for a 'cheap response' to exclusion problems, whose cheapness undercuts the atomist picture.

\footnotetext{
${ }^{2}$ Pun intended. I suspect that colour-exclusion is not a matter of necessity (whether logical, grammatical, or conceptual). Roughly put: I suspect it is merely a biological fact that we do not see colours like we hear musical chords.

Such suspicions need not just result from abstract thought-experiments; we can actually get some concrete sense of what seeing a red-blue colour-chord might be like. Just place two identically sized rectangles, one red and one blue, besides each other, and then go cross-eye, so that your visual images of the two rectangles overlap completely. (This simple approach is described in [1] which also describes a much more controlled experiment.) If an object could look like that through just one eye, why not say it is both red and blue? Or, if you prefer to ascribe colours to patches in the visual field, rather than objects, why not say that patch is both red and blue?

Wittgenstein himself largely describes colour exclusion as necessary ([10]: 6.3751, [11]: 167, [12]: IV.39, VIII.77-78, [13]: 67, 78, 241). However, there is a glimmer of the alternative, in his response ([13]: 79) to Waismann's suggestion that psychologists might empirically investigate colour-exclusion.
} 


\section{A Cheap Response to Exclusion Problems}

To illustrate the 'cheap response', I shall focus on a particular exclusion problem generated by a toy logical space.

Heat Space. Our toy logical space consists of the possible assignments of temperature properties to the points of spacetime. We can think of the points of spacetime as quadruples of real numbers (describing points relative to some coordinate system), and we can think of the temperatures as non-negative real numbers (describing temperature in Kelvins). Any assignment of temperature properties to points of spacetime is possible, subject to two constraints:

(a) Each spacetime point has exactly one temperature property.

(b) The distribution of temperature properties must be continuous.

Whilst still a toy, Heat Space gives rise to an exclusion problem which is a little more interesting than usual. On a minor level: rather than considering (colour-)exclusion for a single object, it considers (temperature-)exclusion for continuum-many objects (the spacetime points) simultaneously. ${ }^{3}$ Much more interestingly: two kinds of exclusion operate in Heat Space. Constraint (b) gives us temperature-exclusion at each point: nothing can be both $17 \mathrm{~K}$ and $18 \mathrm{~K}$ at the same time. But constraint (b) gives us temperature-exclusion across points: whilst you can consistently assign any temperatures to any two points, certain patterns of temperature distribution are ruled out; for example, there cannot be any abrupt local heat spikes. So, Heat Space is certainly a toy, but the restrictions it imposes are not wholly trivial.

Let us now see how the cardinality-considerations of Section 2 apply to Heat Space.

Given the description of Heat Space, the number of possibilities is precisely the number of continuous functions from $\mathbb{R}^{4}$ to $[0, \infty)$. In effect, (b) tells us to consider functions, and (b) tells us that they must be continuous. A little mathematics will convince us that there are exactly $2^{\aleph_{0}}$ such functions. ${ }^{4}$ So, by the cardinalityconsiderations of Section 2, we know that Heat Space can, in principle, be described using the atomist picture. Indeed, we can build a toy model which seems to describe Heat Space, by insisting that there are $\aleph_{0}$ atomic propositions which decompose, under logical analysis, into $\aleph_{0}$ distinct Switches, each concatenated with Flicked. Call this the Flicked-Switch Analysis of Heat Space.

This is a very 'cheap response', in that it brushes aside Heat Space's exclusion problem(s) with a mere gesture at our elementary cardinality-constraint. But, given the availability of this cheap response, we are forced to ask why anyone has ever even thought that exclusion problems raises serious problems for the atomist picture.

Two obvious answers present themselves. First, unlike our toy Heat Space, logical space itself might not be a power of two. In that case, the cardinality-considerations

\footnotetext{
${ }^{3}$ On this point, compare Carruthers's ([3]: 144-7) model.

${ }^{4}$ Let $C$ be the set of continuous functions from $\mathbb{R}^{4}$ to $[0, \infty)$. Constant functions are continuous; so $|C| \geq$ $2^{\aleph_{0}}$. Conversely, if $f, g \in C$ and $f(p)=g(p)$ for all $p \in \mathbb{Q}^{4}$, then $f=g$, since $f$ and $g$ are continuous; but the total number of functions from $\mathbb{Q}^{4}$ to $[0, \infty)$ is $\left(2^{\aleph_{0}}\right)^{\aleph_{0}}=2^{\aleph_{0}}$; so $|C| \leq 2^{\aleph_{0}}$.
} 
of Section 2 would show that the atomist picture is in principle untenable. Second, the Flicked-Switch Analysis, just offered, might simply feel too cheap. As I shall explain in Section 6, both answers are basically correct. Moreover, this explains why we should reject the atomist picture.

\section{Moss's Approach}

First, though, I must embark on a slight detour, and consider Moss's [5] recent claim to have solved the colour-exclusion problem. I want to show that Moss's solution ultimately boils down to the 'cheap response' which I just offered, so that the problems concerning the 'cheap response' are also problems for Moss. But I should add that it is no coincidence that Moss's solution boils down to mine; my paper was prompted by trying to isolate the moving parts of Moss's paper.

\subsection{Moss's Solution}

The core of Moss's response to colour exclusion is the following argument: ${ }^{5}$

(1) 'given an arbitrary set $S$ of propositions, we can always find $2^{\mathfrak{a}}$ (for some $\mathfrak{a}$ ) mutually exclusive propositions such that any member of $S$ is a conjunction of some of them.' ([5]: 846)

(2) 'for any $2^{\mathfrak{a}}$ mutually exclusive propositions, we can find a logically independent propositions such that the mutually exclusive propositions are truthfunctional combinations of them.' ([5]: 845)

So: given an arbitrary set $S$ of propositions, we can find logically independent propositions such that the propositions in $S$ are truth-functional combinations of the logically independent propositions.

The conclusion of this argument amounts to a statement that the atomist picture is tenable in principle. In that sense, it invites comparison with my elementary cardinality-constraint from Section 2. However, I should deal with one immediate difference. In Section 2, I reasoned about logical space; Moss, by contrast, discusses (sets of) propositions. This difference, however, is shallower than it might seem, since Moss 'take[s] propositions to be sets of possible worlds' ([5]: 842). As mentioned in Section 1, nothing turns on calling the possibilities 'possible worlds'. So, in my terminology, Moss's propositions are just regions (rather than points) of logical space. As such, our considerations are commensurable.

I shall revisit this point below. First, I shall consider how Moss defends the premises of her argument. To establish (1), Moss defines a relation on the possible worlds as follows:

$$
w R x \text { iff } p \text { is true at } w \text { iff } p \text { is true at } x, \text { for all } p \in S
$$

\footnotetext{
${ }^{5}$ I have compressed the argument, and numbered the steps. Additionally, in these quotes, and in subsequent quotes from Moss throughout this section, I have silently (but harmlessly) changed several minor points of notation.
} 
She then defines a set, $S^{*}$, as 'the set of equivalence classes of worlds under' $R$ ([5]: 846). Her argument for (1) now proceeds by cases:

(1a) The cardinality of $S^{*}$ is $2^{\mathfrak{a}}$, for some a. In this case, 'we are done'. After all, $S^{*}$ is a set of sets of worlds, and hence (for Moss) a set of propositions, which itself has the required properties.

(1b) The cardinality of $S^{*}$ is not $2^{\mathfrak{a}}$, for any $\mathfrak{a}$. In this case, we should 'repeatedly replace any single member of $S^{*}$ with mutually exclusive propositions whose disjunction [i.e. union] is that member. In this way, we can increase the cardinality of $S^{*}$ until it contains $2^{\mathfrak{a}}$ mutually exclusive propositions.' ([5]: 846)

Below, I shall discuss the assumption which is required to license this 'replacing' in (1b). (Spoiler alert: it is that logical space be a power of two.) But we shall take (1) as established for now.

In defence of (2), Moss works through a particular example, namely, the fact that an object, $O$, can only have one of continuum-many colours. She observes that, just as there are continuum-many colours, so there are 'continuum-many infinitely long sequences of 0's and 1's.' So Moss 'fix[es] a bijection between these sets, a mapping from possible colors of $O$ to infinite binary strings.' Call this bijection $f$. Then Moss asks us to consider, for each natural number $i$, the proposition: ${ }^{6}$

$F_{i}$ : that $f$ maps $O$ 's colour to a string with a 1 in the $i^{\text {th }}$ place

These propositions are logically independent from one another. Moreover, for each subset, $C$, of the natural numbers, the infinite conjunction $\bigwedge_{i \in C} F_{i} \wedge \bigwedge_{i \notin C} \neg F_{i}$ pins down a unique colour for $O$. So 'each ordinary language color proposition [concerning $O$ ] is a truth-functional combination of the $F_{i}$ propositions' ([5]: 845). Moss then generalises from this observation, to arrive at (2), by noting that the case of $O$ 's colour was just an illustrative example in which $\mathfrak{a}=\aleph_{0}$.

This completes my summary of Moss's argument. In the remainder of this section, I shall try to show that the key take-away from Moss's solution is precisely the cardinality-constraint of Section 2, with its concomitant 'cheap response' to exclusion problems.

\subsection{On Ordinary Language}

I shall start by revisiting the difference I raised at the start of this section, concerning Moss's focus on propositions. She does this, because she wants to deliver on the claim that 'we can completely analyze all ordinary language propositions' ([5]: 841). In this regard, my approach diverges from hers, since I did not mention 'ordinary language' anywhere in Sections 1-3. This difference, however, is rather superficial.

Moss's approach certainly seems to be quite tightly connected with ordinary language. Indeed, in defending (2), Moss may seem to have defined each $F_{i}$ within

\footnotetext{
${ }^{6}$ This fixes (what I take to be) a small typesetting error in Moss's paper. I have also incorporated the bijection $f$ explicitly into the (statement of) each proposition; this is both strictly-speaking necessary and more than mere pedantry (see below).
} 
ordinary language. But appearances here are misleading. In defining each $F_{i}$, Moss appealed to a bijection, $f$. But she did not explicitly specify $f$. Instead, she 'fixed' it, as the mathematicians say. And she is forced to 'fix' it, for English contains no names for particular maps from colours to infinite binary strings, nor the means for defining any explicitly. Otherwise put: we know that the $F_{i}$ s exist, but we have no means to express them in ordinary language.

The separation between ordinary language and Moss's solution grows deeper when we consider Moss's discussion of case (1b). We may well want to 'repeatedly replace any single member of $S^{*}$ with mutually exclusive propositions whose disjunction is that member'. But there is no guarantee that ordinary language provides us with the words for such mutually exclusive propositions. Again: there is no guarantee that ordinary language allows us to express the atomic propositions which Moss ends up with.

Indeed, the only real bridge which Moss builds to 'ordinary language propositions' is in her decision to 'take propositions to be sets of worlds' ([5]: 842). But this just returns me to the point I made at the start of the section. Nothing prevents me from taking propositions - ordinary language, or otherwise - to be sets of possibilities, i.e. regions of logical space. ${ }^{7}$

In sum: despite initial appearances, Moss's approach gives us no closer relationship to ordinary language than my own.

\subsection{On Arbitrary Propositions}

There remains a difference, in that Moss allows us to start with arbitrary sets of propositions-i.e. sets of regions of logical space-whereas I leap straight into logical space itself.

In fact, starting with arbitrary sets of propositions can lead to oddities. Let $p$ be the proposition that the present King of France is bald, and let $S=\{p\}$. Following Moss's argument for (1), we form $S^{*}$ from $S$. This partitions the possible universe into two sets: the set of worlds where $p$ is true, and the set of worlds where $p$ is false. Since the cardinality of $S^{*}$ is 2 , we are in case (1a), and 'we are done'. We now apply (2) to $S^{*}$, and so find exactly one (logically independent!) proposition such that the propositions in $S^{*}$ are truth-functional combinations of it. Plainly, that proposition is $p$ itself. And this encourages us to think of $p$ as an atomic proposition. But, of course, $p$ is not atomic.

To get closer to the genuinely atomic propositions, we must start with a richer set than $\{p\}$. There is no better set in this regard than the set of all propositions. Call this set $P$ and, as before, form $P^{*}$ from $P$. Following Moss, we now reason by cases. In case (1a), there are $2^{\mathfrak{a}}$ propositions in $P^{*}$, for some $\mathfrak{a}$, and 'we are done'. But in case (1b) we run into trouble. ${ }^{8}$ We are supposed to 'repeatedly replace any single member of $P^{*}$ with mutually exclusive propositions whose disjunction is that

\footnotetext{
${ }^{7}$ Indeed, this is quite Tractarian; (see [10]: 4.4-4.431). On this picture, if there are $\mathfrak{a}$ atomic propositions, then there are $2^{\left(2^{\mathfrak{a}}\right)}$ propositions, i.e. $2^{\left(2^{\mathfrak{a}}\right)}$ sets of possible worlds.

${ }^{8} \mathrm{My}$ argument in this paragraph assumes — as Moss surely does — that worlds are identical iff exactly the same propositions are true in them.
} 
member'. Unfortunately, we cannot do this. By assumption, $P$ was the set of all propositions, so that $P^{*}$ is the finest equivalence class on the possible universe. In short, for Moss's approach to work, she must simply assume that case (1b) cannot arise here. Otherwise put: she must assume that the cardinality of $P^{*}$ is $2^{\mathfrak{a}}$ for some a. Since a moment's reflection will convince us that $P^{*}$ is the set of all singletons of possible worlds, we can restate the point again as follows: to defend (1), Moss must assume that logical space is a power of two.

Now, for reasons in precisely this ballpark, Moss herself suggests that we should assume that 'the number of worlds is a power of 2'. This is all to the good. However, she describes this as a 'weak assumption' which 'suffices' for her defence of the atomist picture ([5]: 846, 847). Here, I demure. The point of Section 2 is that this supposedly 'weak assumption' is not merely sufficient for the in-principle-tenability of the atomist picture, but also necessary for it. (Moreover, since the assumption is logically equivalent to the in-principle-tenability of the atomist picture, we might well question whether it really is 'weak'; I return to this in Section 6.)

The main point of all this is as follows. Allowing arbitrary sets of propositions leads to odd verdicts concerning the atomic propositions. And, when we strip matters back to the key case - the set of all propositions - the only point to extract is the simple fact that logical space can be described by the atomist picture if and only if logical space is a power of two. That, of course, was the point of Section 2.

\subsection{On Colours, Functions, and Strings}

The final point I want to emphasise is that Moss's approach does not give us any clue about the correct logical analysis of the atomic propositions. ${ }^{9}$

There are some obvious similarities between my considerations in Section 2 and Moss's discussion of $O$ 's colour. ${ }^{10}$ Indeed, Moss's $F_{i}$ s are quite similar to the atomic propositions I provided in my Flicked-Switch Analysis of Heat Space. Just think of the $\aleph_{0}$ Switches as arranged in an $\omega$-sequence, and write a ' 1 ' beneath a flicked switch and a ' 0 ' beneath a non-flicked switch.

However, I want to emphasise that Moss's $F_{i}$ s do not provide us with a good logical analysis of the propositions involved in colour-exclusion. In a sense this is obvious: her $F_{i}$ s were given whilst considering a toy example which arose just by considering the set of propositions stating $O$ 's possible colours. But the point I want to make is rather more general, namely: atomic propositions cannot consist of anything like (names for) colours and functions and strings. ${ }^{11}$ We can see this in two ways.

First Argument. Moss used the map $f$ from colours to strings. Let us consider a different map, $g$, as follows: where $f$ maps the colour $c$ to the string $s$, let $g$ map $c$ to $s$ 's 'binary opposite', i.e. to the string which has a 1 wherever $s$ has a 0 and vice versa. Using $g$, we then consider, for each natural number $i$, the proposition:

\footnotetext{
${ }^{9}$ But nor does my own, as we shall see in Section 6.

${ }^{10}$ And also with Carruthers ([3]: 144-7).

${ }^{11}$ Moss ([5]: 848-50) discusses a related but different problem, namely, that the propositions she generates via (1) will likely contain apparent names for logical constants, such as 'or'. Her response to that problem, in brief, is that simplicity is relative to a language. This does not affect my point.
} 
$G_{i}$ : that $g$ maps $O$ 's colour to a string with a 1 in the $i^{\text {th }}$ place

The $G_{i}$ s are as logically independent from each other as were the $F_{i}$ s. However, there are dependencies between the $F_{i}$ s and the $G_{i}$ s: since $f$ and $g$ map the same colour to 'opposite' strings, exactly one of $F_{i}$ and $G_{i}$ is true (for each $i$ ), regardless of $O$ 's colour. This is, of course, just another exclusion problem. But no exclusion problems can remain in the final analysis.

Second Argument. ${ }^{12}$ Let us assume that colours are simple. ${ }^{13}$ Nevertheless, a term of the form ' $f(c)$ ' - which is supposed to pick out the string which $f$ sends $c$ tois obviously complex. So, by Simples-Recombination, ' $f(c)$ ' cannot appear in an atomic proposition. A good Tractarian must therefore replace the occurrence of the map $f$, in each $F_{i}$, with some propositional function. But, since we are assuming that the colours are simples, it follows from Simples-Recombination (again) that there is some world, $w$, where all the colours satisfy exactly the same propositional functions as each other. Accordingly, in w, every colour is 'mapped' to the same string. As such, we cannot regard $O$ 's colour in $w$ as a truth-functional combination of the $F_{i}$ s. ${ }^{14}$

The upshot of both arguments is that the atomic propositions cannot consist of anything like (names for) colours and functions and strings. Once we have appreciated this point, though, we see that Moss's discussion of colours and functions and strings is just a vivid illustration of her point (2), which provides us with no guide at all to the logical form of the atomic propositions. The key point to extract-once again-is just an observation about cardinality.

\section{Abandoning Atomic-Independence}

Returning from my discussion of Moss, here is the story of the paper so far. We saw in Section 2 that the in-principle-tenability of the atomist picture reduces to an elementary cardinality-constraint. Moreover, this provides us with a 'cheap response' to exclusion problems, as I showed by applying the cardinality-constraint of Section 2 to Heat Space in Section 4. The upshot was a particular toy analysis- the Flicked-Switch Analysis-where $\aleph_{0}$ atomic propositions generate the $2^{\aleph_{0}}$ possible temperature distributions in Heat Space. To close the paper, I want to explain how unsatisfying the Flicked-Switch Analysis is, and thereby to vindicate Wittgenstein's rejection of Atomic-Independence.

In the Flicked-Switch Analysis of Heat Space, we have a bijection between configurations of Switches (flicked or not) and the possibilities in Heat Space. So, we know that if you change whether a particular switch is flicked or not, you change the way in which temperatures are assigned to points. But there is no a priori constraint on how these changes occur. Starting from some given possibility: by 'toggling' Switch 1, everything which was hot might become cold; by then 'toggling' Switch 2, an

\footnotetext{
${ }^{12}$ Thanks to Rob Trueman for this observation; for related discussion, see Trueman [8]: 296.

${ }^{13}$ See Canfield [2].

${ }^{14}$ And of course the same issue arises for binary strings, since strings are functions from the natural numbers to 0 and 1 .
} 
unmeasurable collection of points might become freezing; and by 'toggling' Switch 1 again, the world may turn uniformly balmly. Or not. The relationship between the states of the Switches, on the one hand, and the temperatures at each point, on the other, is entirely opaque. ${ }^{15}$

This is one reason to be dissatisfied with the Flicked-Switch Analysis. A second reason is that genuinely alternative logical analyses are available. We showed that Heat Space comprises $2^{\aleph_{0}}$ possibilities. It follows that, if the atomist picture is to be applied to Heat Space, there must be $\aleph_{0}$ atomic propositions. However, there is no reason to think that they must decompose, under logical analysis, into Flicked Switches. Instead, the atomic propositions might decompose according to the following Yin-Yang Analysis: there are $\aleph_{0}$ Yins and $\aleph_{0}$ Yangs, and the atomic propositions are all and only the concatenations of (the name of) any Yin with (the name of) any Yang.

The Yin-Yang Analysis is consistent with the atomist picture. To show that it satisfies Simples-Recombination, we just need to specify that the Yins and the Yangs are simples of different logical types. Indeed, we can regard the Yins as first-order objects and the Yangs as one-place properties, or vice versa (cf. Ramsey [8]). Moreover, since $\aleph_{0} \cdot \aleph_{0}=\aleph_{0}$, the Yin-Yang Analysis also yields $\aleph_{0}$ atomic propositions. But the Yin-Yang and the Flicked-Switch analyses are genuinely different. According to the Flicked-Switch Analysis, every Switch occurs in exactly one atomic proposition, and the property Flicked occurs in every atomic proposition. By contrast, according to the Yin-Yang Analysis, each Yin and each Yang both occur in, and are absent from, exactly $\aleph_{0}$ atomic propositions. (And inevitably, just as we had no idea how flicking Switches will affect the temperature assignment, so we have no idea how coupling or decoupling Yins and Yangs affects temperatures.)

We need not stop at the Yin-Yang Analysis: any way to generate $\aleph_{0}$ atomic propositions from simples will provide us with a toy analysis of Heat Space. For example, we can turn the Yin-Yang Analysis into the Aristophanean Analysis, by treating the atomic propositions as arbitrary concatenations of any (distinct) Yins and Yangs. Alternatively, here is a schema for generating infinitely many rival analysis: let there be $\aleph_{0}$ different kinds of simples; let there be $\aleph_{0}$ instances of each kind; and let the atomic propositions be all and only the concatenations of names for $n$ distinct entities drawn from exactly $m$ different kinds (with $0<m \leq n<\omega$ ).

I could keep going, but the examples given already suffice to raise a pressing question: What is the correct logical analysis of Heat Space, and how does that analysis relate to temperature distributions?

There seems to be no a priori way to answer the pressing question. Nothing more is given, a priori, by the specification of Heat Space and the desire to apply the atomist picture to it, than that there must be $\aleph_{0}$ atomic propositions. This both underdetermines the logical form of the atomic propositions, and tells us nothing about their relationship to temperature distributions. In principle, perhaps, maybe, some further a priori constraint could be deployed, which provides us with more information. But I, for one, just cannot see what it would look like.

\footnotetext{
${ }^{15}$ This is connected with the point, above, that Moss simply 'fixed' some bijection, $f$.
} 
Equally, there seems to be no a posteriori way to answer the pressing question. ${ }^{16}$ To have an a posteriori route to the correct analysis of logical space, physics, or ordinary language, would have to be pushing towards a language within which exclusion problems did not arise. Unfortunately, as Wittgenstein himself noted, we seem unavoidably to use numbers (or other devices of gradation) in representing propositions, and there are exclusion problems whenever there are gradations ([11]: 165-7).

So we seem unable to answer the pressing question. And this point generalises beyond the toy example of Heat Space, to logical space itself. Proponents of the atomist picture seem forced to accept that we can neither determine the correct analysis of the atomic propositions, nor say in any detail how their constituents relate to the 'manifest' possibilities. Consequently, proponents of the atomist picture seem forced to embrace Ramsey's ([8]: 417) conclusion 'that we know and can know nothing whatever about the forms of atomic propositions'. But, as Wittgenstein ([13]: 182-4) himself came to stress: it is one thing to think that we do not know the forms of atomic propositions; it is vastly less palatable to suggest that we cannot know anything about them. ${ }^{17}$

To ice the unpalatable cake, there remains the fact that the atomist picture demands that logical space be a power of two. If we are not already committed to the correctness of the atomist picture, why on earth would we have any beliefs about the cardinality of logical space? A priori reasons here seem thin on the ground. A posteriori evidence might just about be available, since a scientific theory might predict that logical space is a power of two-consider again the case of Heat Space-but there is no real reason to be optimistic on that front.

All told, then, Atomic-Independence comes across as a dogma with unpalatable consequences. Ultimately, the issue is not that we can find no solution to the exclusion problem. The issue is rather as follows: we have no guarantee that there is a solution to the exclusion problem; but if there are any, then there are too many. ${ }^{18}$

Open Access This article is distributed under the terms of the Creative Commons Attribution 4.0 International License (http://creativecommons.org/licenses/by/4.0/), which permits unrestricted use, distribution, and reproduction in any medium, provided you give appropriate credit to the original author(s) and the source, provide a link to the Creative Commons license, and indicate if changes were made.

\section{References}

1. Billock, V.A., \& Tsou, B.H. (2010). Seeing forbidden colors. In Scientific American 302.2 (pp. 72-7).

2. Canfield, J.V. (1976). Tractatus Objects. In Philosophia 6.1 (pp. 81-99).

\footnotetext{
${ }^{16}$ Wittgenstein ([10]: 5.55-5.551) initially seemed to reject a posteriori routes to logical form; but later ([11]: 163-4) became more receptive.

${ }^{17}$ See also Wittgenstein ([10]: 5.55, 5.5571) and Medina ([4]: 371, 377-8, 384).

${ }^{18}$ I wrote this paper during a period of research leave which was funded by a Philip Leverhulme Prize (awarded by the Leverhulme Trust, PLP-2014-140). For comments and discussion on this paper, thanks to Luke Cash, Sarah Moss, Fredrik Nyseth, Michael Potter, Robert Trueman, and an anonymous referee for this journal.
} 
3. Carruthers, P. (1990). The Metaphysics of the Tractatus. Cambridge: Cambridge University Press.

4. Medina, J. (2003). Deflationism and the true colours of necessity in Wittgenstein's Tractatus. In Dialectica 57.4 (pp. 357-85).

5. Moss, S. (2012). Solving the Color Incompatibility Problem. In Journal of Philosophical Logic 41.5 (pp. 841-51).

6. Potter, M. (2009). Wittgenstein's Notes on Logic. Oxford: Oxford University Press.

7. Ramsey, F.P. (1923). Critical Notice of Wittgenstein's Tractatus. In Mind 32.128 (pp. 465-78).

8. Ramsey, F.P. (1925). Universals. In Mind 34.136 (pp. 401-17).

9. Trueman, R. (2011). Propositional Functions in Extension. In Theoria 77 (pp. 292-311).

10. Wittgenstein, L. (1921). Tractatus Logico-Philosophicus. Trans, In Pears, D.F., \& McGuinness, B.F. (Eds.) Revised 2002 edition, from Routledge.

11. Wittgenstein, L. (1929). Some Remarks on Logical Form. In Proceedings of the Aristotelian Society, Supplementary, (Vol. 9 pp. 162-71).

12. Wittgenstein, L. (1964). In Rhees, R. (Ed.) Philosophische Bemerkungen. Oxford: Basil Blackwell.

13. Wittgenstein, L. (1979). In McGuinness, B. (Ed.) Wittgenstein and the Vienna Circle: Conversations recorded by Friedrich Waismann. Oxford: Basil Blackwell. 